\title{
An improved reverse dot hybridization for simple and rapid detection of adefovir dipivoxil-resistant hepatitis $B$ virus
}

\author{
Y. Hu${ }^{1}$, W.L. Zhang ${ }^{1}$, S.L. Xie ${ }^{1}$, Y. Zhao ${ }^{2}$, J.L. Hu ${ }^{1}$, X.F. Cai ${ }^{1}$, G.Q. Lai ${ }^{1}$ \\ and A.L. Huang ${ }^{1}$ \\ ${ }^{1}$ Key Laboratory of Molecular Infectious Diseases, Ministry of Education, \\ Chongqing Medical University, Chongqing, China \\ ${ }^{2}$ Pediatric Research Institute, \\ Ministry of Education Key Laboratory of Child Development and Disorders, \\ Children's Hospital, Chongqing Medical University, Chongqing, China \\ Corresponding author: A.L. Huang \\ E-mail: ahuang1964@163.com
}

Genet. Mol. Res. 11 (1): 53-60 (2012)

Received August 31, 2011

Accepted October 10, 2011

Published January 9, 2012

DOI http://dx.doi.org/10.4238/2012.January.9.6

\begin{abstract}
Early detection of adefovir dipivoxil-resistant mutants during long-term treatment of chronic hepatitis B virus (HBV) infection with this drug is of great clinical importance. We developed an improved reverse dot hybridization test for simple and rapid detection of the rtA181V/T and rtN236T mutations associated with adefovir dipivoxil resistance in chronic hepatitis B patients. Probes were designed for genotypes $\mathrm{B}, \mathrm{C}$, and $\mathrm{D}$ of this resistance characteristic; a total of 70 clinical samples were analyzed with this improved reverse dot hybridization assay. Its usefulness was validated by comparing with sequencing data. Discordant results were confirmed by subclone sequencing. This reverse dot hybridization assay was sufficiently sensitive to detect $10^{3}$ copies $/ \mathrm{mL}$; it also detected adefovir dipivoxilresistant mutant strains when they comprised more than $5 \%$ of a mixed virus population. This reverse dot hybridization array correctly identified adefovir dipivoxil-resistant mutants; it had high concordance
\end{abstract}


(98.5\%) with direct sequencing data. There was no clear relationship between the HBV genotype and the development of adefovir dipivoxilresistant mutants. This reverse dot hybridization assay proved to be simple and rapid for detection of rtA181V/T and rtN236T mutations associated with resistance to adefovir dipivoxil.

Key words: Hepatitis B virus; Reverse dot hybridization; Mutant; Adefovir dipivoxil

\section{INTRODUCTION}

Hepatitis B virus (HBV) is an enveloped virus with a double-stranded DNA genome of $3200 \mathrm{bp}$. HBV infection is still one of the most serious and prevalent health problems. Current reports suggest that it causes $30 \%$ of cirrhosis and approximately $50 \%$ of hepatocellular carcinoma globally (Perz et al., 2006). Nucleoside analogs such as adefovir dipivoxil (ADV) are approved as first-line treatment antiviral drugs. Adefovir dipivoxil is an oral prodrug of an acyclic monophosphate adenine analog, and most clinical studies have shown that ADV could effectively reduce serum HBV-DNA levels and improve serum alanine aminotransferase (ALT) levels as well as liver histology (Hadziyannis et al., 2006; Delaney, 2007). However, as with several other analogues, long-term ADV monotherapy or combination therapy with lamivudine results in complete suppression of viral replication and is associated with the emergence of resistant mutants (Dai et al., 2007; Santantonio et al., 2009). Many reports have confirmed that $\mathrm{rtN236T}$ and $\mathrm{rtA} 181 \mathrm{~V} / \mathrm{T}$ mutations, at loci in the reverse-transcriptase (RT) domain of HBV polymerase, are associated with ADV failure (Aloman and Wands, 2003; Villet et al., 2008). The sensitive detection of such mutations before or early in treatment could assist in optimizing antiviral treatment. Therefore, developing a sensitive, specific, accurate, and easy to perform method for the detection of ADV-resistant HBV is of great clinical importance.

A number of techniques are currently available for detecting HBV mutants. Direct sequencing of HBV DNA is regarded as the gold standard for detecting the emergence of ADV-resistant HBV mutant, but it is a time-consuming and laborious method. In addition, this method only detects mutant virus when it comprises at least $25 \%$ of the total virus population (Lok et al., 2007). Other molecular methods, such as PCR-restriction fragment length polymorphism (RFLP) analysis, peptide nucleic acid-mediated PCR clamping (Ohishi et al., 2004), ligase detection reaction (Wang et al., 2009), microarray (Tran et al., 2006), and mass spectrometric analysis (Luan et al., 2009), are highly reliable but time-consuming, requiring a skillful technician to perform them.

The reverse dot blot (RDB) method can simultaneously detect many point mutations, and is currently in use for identification of disease related to gene mutations such as Wilson's disease (Lovicu et al., 2003). LIPA, a commercial assay kit based on reverse hybridization is a very sensitive and specific assay for the detection of ADV mutants (Osiowy et al., 2006). However, the strips have many lines to represent ADV mutants, making it more complicated to determine. As the genotypes in China are mainly B, C and D, we established an improved RDB system for the simple, rapid and accurate detection of the rtA181V/T and $\mathrm{rtN} 236 \mathrm{~T} \mathrm{mu-}$ tations. The diagnostic reliability of this assay was evaluated in 70 samples by using direct sequencing in a blind analysis. 


\section{MATERIAL AND METHODS}

\section{Plasmids}

For standard assay quantification, HBV plasmids containing the RT domain were constructed as follows. Clinical samples of wild-type or ADV mutants $(181 \mathrm{~V}+236 \mathrm{~T}$ or $181 \mathrm{~T}+236 \mathrm{~T}$ ) from the First Affiliated Hospital were amplified with the primer pair HBV RTFP: 5'-GAAGACTGGGGACCCTGTACCGAAC-3' and HBV RTRP: 5'-TTGCCGGGCAAC GGGGTAAAGGTTC-3', and cloned into the pMD18-T Vector (Takara, Dalian, China) according to manufacturer instructions. Finally, these clones were confirmed by direct sequencing.

\section{Patient samples}

A total of 140 samples from 70 chronic HBV patients undergoing treatment with ADV monotherapy were collected for analysis. Samples collected at baseline and at 48 weeks were available. All of them met the following criteria: a) presence of HBeAg and HBV DNA in serum, and an elevation of serum ALT at least 2 times higher than the normal value during the year prior to the initiation of the study; b) hepatitis B surface antigen (HBsAg) positive in serum for at least six months. The genotypes and concentration of HBV DNA in these samples had been determined by our GQ-PCR assay (Zhao et al., 2010). This study was approved by the ethical committee of the first affiliated hospital of Chongqing Medical University, and informed consent was obtained from each patient before enrollment.

\section{Extraction of HBV DNA from serum samples and PCR conditions}

HBV DNA was isolated from $200 \mu \mathrm{L}$ serum by using the commercially available AquaSPIN Liquid Sample Total NA Isolation kit (Biowatson, Shanghai, China). The target DNA used for hybridization was amplified by semi-nested PCR. The nested primers (Invitrogen, Shanghai, China) are listed in Table 1. The forward primers were labeled with phosphoric acid at the 5' end, while the reverse primer was labeled with biotin at the $5^{\prime}$ end. The PCR conditions included 4 min at $94^{\circ} \mathrm{C} ; 35$ cycles of $40 \mathrm{~s}$ at $94^{\circ} \mathrm{C}, 40 \mathrm{~s}$ at $56^{\circ} \mathrm{C}$, and $60 \mathrm{~s} 72^{\circ} \mathrm{C}$, and $10 \mathrm{~min}$ at $72^{\circ} \mathrm{C}$. The PCR products were $690 \mathrm{bp}$, analyzed on a $1.2 \%$ agarose gel, and visualized by staining with ethidium bromide. If not, $1 \mu \mathrm{L}$ of the product from the first PCR was used for the second PCR.

\begin{tabular}{lll}
\multicolumn{2}{l}{ Table 1. Primers and probes used in our RDB protocol. } & \\
\hline Primer or probe & Sequence (5'-3') & Comments \\
\hline Primers & & \\
$420 \mathrm{FP}$ & 5'-ATCCTGCTGCTATGCCTCAT-3' & Labeled with phosphoric acid at 5' end \\
$470 \mathrm{FP}$ & 5'-TGTTGCCCGTTTGTCCTCTA-3' & Labeled with phosphoric acid at 5' end \\
$1110 \mathrm{RP}$ & 5'-TGGCGAGAAAGTRAAAGCCTG-3' & Labeled with biotin at 5' end \\
Probes & & \\
A 181 & 5'-TCTCTTGGCTCAGTTTAC-3' & \\
$181 \mathrm{~V}$ & 5'-TCTCTTGGTTCAGTTTAC-3' & \\
$181 \mathrm{~T}$ & 5'-TCTCTTGACTCAGTTTAC-3' & \\
N236 & 5'-GGTATACATTTAAACCC-3' & \\
Ctrl 690 & 5'-GGTATACATTTAACCCC-3' & Amplification control probe \\
\hline
\end{tabular}

Genetics and Molecular Research 11 (1): 53-60 (2012) 


\section{Design of target-specific probes, $\mathrm{CC}$ probes, and $\mathrm{AC}$ probes}

A total of 1887 sequences of HBV accepted by GenBank (genotype B: 487; genotype C: 1030; genotype D: 370) were collected and aligned using the CLUSTAL X program, and then edited by the GENEDOC program. Five target-specific probes, based on the constructed HBV genotype B, C and D map, were designed to detect ADV-resistant HBV. The probes were designed based on the percent of GC content, probe length and location (Table 1). The color control (Ctrl) represented the validity of the hybridization process, and the amplification control (Ttrl) represented the validity of PCR amplification.

\section{Hybridization and color development}

The control and specific probes were applied to each spot of nitrocellulose (NC) membranes, and then fixed by UV cross-linking or heat at $120^{\circ} \mathrm{C}$ for $30 \mathrm{~min}$. To obtain the single strand for reverse dot blot analysis, exonuclease was added to $10 \mu \mathrm{L}$ PCR product to digest strand (+) with phosphoric acid group of the PCR products. The biotinylated-single strand (-) was then hybridized with the NC membrane containing the normal (WT) and mutant (MT) probes in $2 \mathrm{~mL}$ hybridization buffer ( $2 \mathrm{X} \mathrm{SSC}, 0.1 \%$ sodium dodecyl sulfate) ( $1 \mathrm{X} \mathrm{SSC}=0.3$ $\mathrm{M} \mathrm{NaCl}, 0.03 \mathrm{M}$ sodium citrate) at $53^{\circ} \mathrm{C}$ for $30 \mathrm{~min}$. Membrane strips were then washed in $0.5 \mathrm{X} \mathrm{SSC}, 0.1 \% \mathrm{SDS}$ at $53^{\circ} \mathrm{C}$ for $30 \mathrm{~min}$. The strips were then reacted at room temperature for 30 min with streptavidin horse-radish peroxidase (Roche, Mannheim, Germany) in TTBS, followed by washes $(2 \mathrm{~min} \times 2)$ in $2 \mathrm{X} \mathrm{SSC}$ and $0.1 \% \mathrm{SDS}$ and $1 \mathrm{~min}$ in $0.05 \mathrm{M}$ Tris, pH 9.5, $5 \mathrm{mM} \mathrm{MgCl}{ }_{2}, 0.1 \mathrm{M} \mathrm{NaCl}$. The color was then developed in the dark for about 30 min with NBT/BCIP until the corresponding color appeared.

\section{Sequencing of clinical samples}

To evaluate the efficiency of this improved RDB array, the PCR products of clinical samples used for direct sequencing were purified by the High PCR Product Wizard kit (Qiagen $\mathrm{GmbH}$, Hilden, Germany) and sequenced with the ABI Prism 3100 Genetic Analyzer (Applied Biosystems, CA, USA) in our laboratory. For subclone sequencing, the PCR products were cloned into the pMD18-T Vector according to manufacturer instructions, and 30 clones of each sample were then selected for sequencing.

\section{RESULTS}

\section{Immobilization of the oligonucleotides}

Instead of the traditional covalent attachment via an amino linker, immobilization of the oligonucleotides to the membrane by terminal poly-T tailing was preferred. In order to investigate the effect of different lengths of terminal poly-T tailing on sensitivity signals, different length probes of poly-T tailing were chemically synthesized and directly bound to $\mathrm{NC}$ membranes by UV cross-linking or heat at $120^{\circ} \mathrm{C}$. As shown in Figure 1A, the increase in signal intensity was accompanied by a gradual increase in poly-T length of probes at the N236T site, which indicated the feasibility of increasing of probe length to improve signal strength. 
However, nonspecific hybridization dots appeared at 25T tailing. Similar results were obtained with probes at the $\mathrm{A} 181 \mathrm{~V} / \mathrm{T}$ site (data not shown). Therefore, 20T tailing was chosen for immobilization on NC membranes.

A

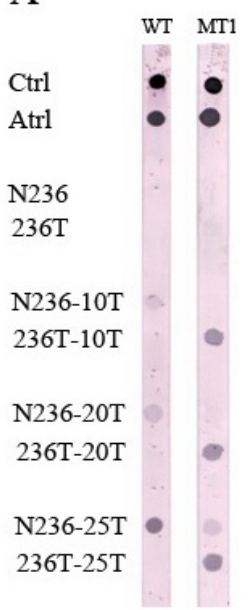

\section{B}

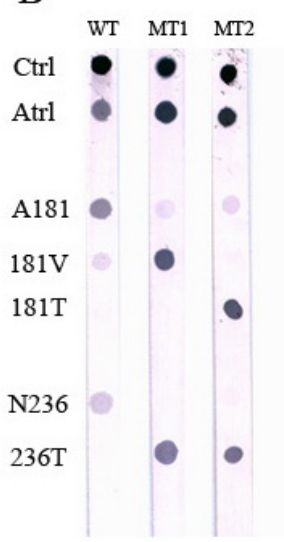

$47^{\circ} \mathrm{C}$

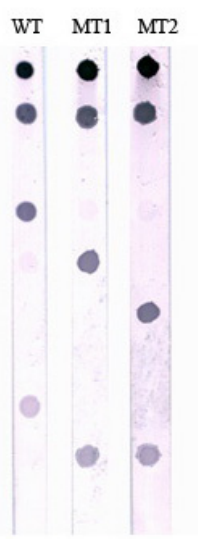

$50^{\circ} \mathrm{C}$

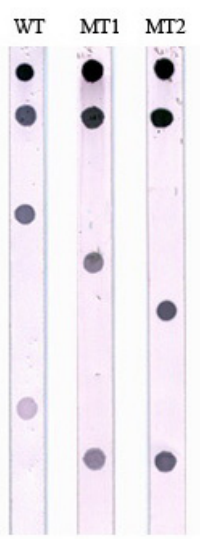

$53^{\circ} \mathrm{C}$

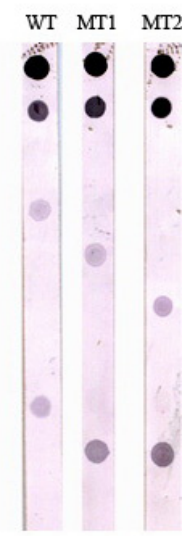

$56^{\circ} \mathrm{C}$

Figure 1. Optimization of hybridization profiles of RDB assay. A. Optimization of probe length. B. Optimization of hybridization temperatures. Ctrl $=$ color control; Ttrl = amplification control; WT $=$ wild-type plasmid $(\mathrm{A} 181+\mathrm{N} 236) ; \mathrm{MT} 1$ = mutant-type plasmid $(181 \mathrm{~V}+\mathrm{N} 236) ; \mathrm{MT} 2$ = mutant-type plasmid $(181 \mathrm{~T}+\mathrm{N} 236)$.

\section{Standardization of optimal hybridization conditions}

The optimal probe concentration was first investigated by 2-fold serial dilutions of each individual probe, starting at a concentration of $0.25 \mu \mathrm{M}$ (Figure 2). The increase in signal was accompanied by a remarkable increase between 0.25 and $5 \mu \mathrm{M}$. At $10 \mu \mathrm{M}$, the signal intensity was only $4 \%$ higher than that at $5 \mu \mathrm{M}$, indicating $5 \mu \mathrm{M}$ as the optimal concentration for hybridization. Furthermore, a strong signal but with nonspecific hybridization dots appeared at $47^{\circ}$ and $50^{\circ} \mathrm{C}$, while a weak positive dot hybridization appeared at $56^{\circ} \mathrm{C}$ (Figure $1 \mathrm{~B}$ ). This indicated that $53^{\circ} \mathrm{C}$ was the optimal temperature for hybridization to distinguish wild and mutant type.

\section{Sensitivity and limit of detection of RDB assay}

The detection limit of our improved RDB assay was determined by 10 -fold serial dilutions of a mixture of standard wild-type and mutant plasmids $\left(10^{2}\right.$ to $10^{8}$ copies $\left./ \mathrm{mL}\right)$. The products of the $10^{3}$ copies $/ \mathrm{mL}$ amplified by semi-nested PCR displayed the positive signal determined by this assay, indicating this viral load as the lower limit of detection. Furthermore, when the two plasmids were mixed at 100:0, 95:5, 90:10 ratios (WT:MT1 or WT:MT2), this assay could detect the minor population present in $5 \%$ of the total viral load (data not shown), suggesting that RDB method is superior to direct sequencing for detecting mutant strains. 


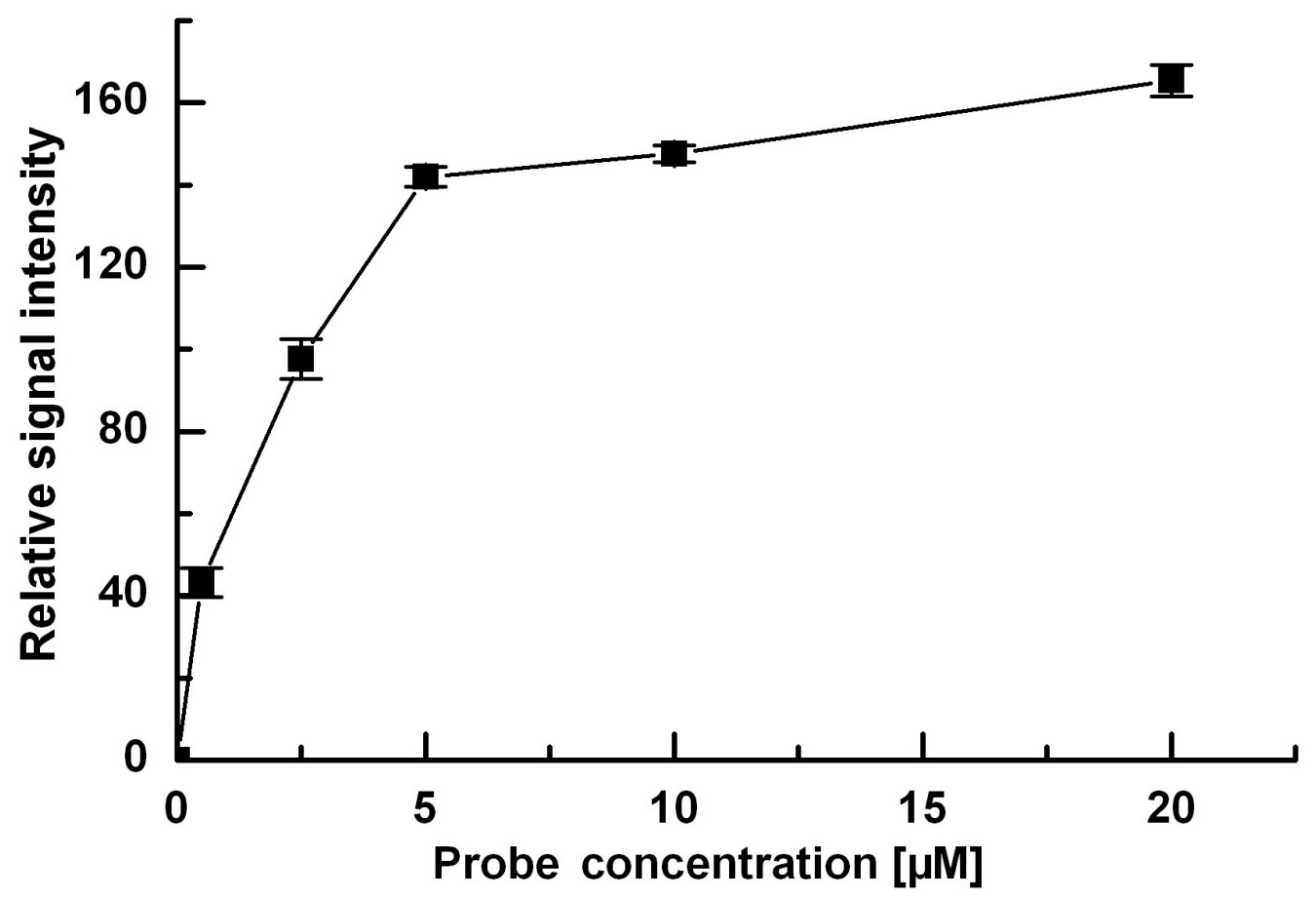

Figure 2. Optimization of probe concentration of RDB assay. Values are shown as averages $\pm \mathrm{SE}$ of three independent experiments.

\section{Comparison of RDB array and direct sequencing}

Next, we investigated $140 \mathrm{HBV}$ clinical samples from 70 patients using our RDB assay and direct sequencing with blind analysis. A total of 136 samples were positively amplified by semi-nested PCR, except for 2 samples that had viral loads less than 1000 copies/ $\mathrm{mL}$ confirmed by our GQ-PCR assay. Samples at baseline were found to be wild-type. After receiving 48 weeks of ADV monotherapy, our RDB assay detected the rtA181V and rtN236T mutations in 8 patients, while direct sequencing only confirmed this in 6 patients. Therefore, complete concordance of our RDB assays is $98.53 \%$ (134/136) with direct sequencing (Table 2). Furthermore, discrepancies in these 2 samples between the two methods were confirmed by subclone sequencing.

\begin{tabular}{lrc}
\multicolumn{2}{c}{ Table 2. Concordant results obtained with RDB assay and direct sequencing of 136 serum samples. } \\
\hline Mutation type & RDB & Direct sequence analysis \\
\hline Wild-type & 128 & 130 \\
rtA181V/T & 0 & 0 \\
rtN236T & 4 & 4 \\
rtA181V/T+rtN236T & 4 & 2 \\
Total & 136 & 136 \\
\hline
\end{tabular}


Last, baseline HBV genotype characterization of the 68 patients determined by our GQ-PCR assay was as follows: 43 samples $(63.23 \%)$ were infected with HBV genotype B alone, only $6(8.82 \%)$ were infected with genotype C alone, and 19 (27.94\%) were infected with both genotypes B and C. Obviously, there was no association observed between genotype and ADV mutation pattern: among the 8 patients with ADV-resistance, 2 were genotype $\mathrm{B}$ and 6 were mixed genotypes $(\mathrm{B} / \mathrm{C})$ at baseline, as shown in Table 3 . However, it is worth noting that the mixed genotype $(\mathrm{B} / \mathrm{C})$ in the 5 patients $(5 / 8)$ had switched to genotype $\mathrm{B}$ alone after 1 year of treatment.

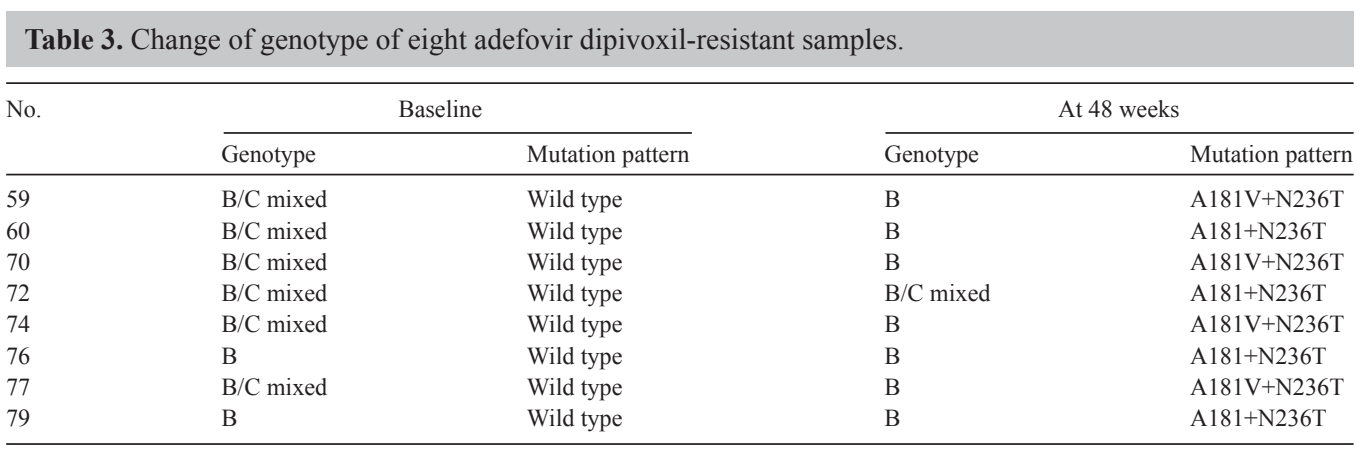

\section{DISCUSSION}

It is well known that long-term therapy with ADV leads to treatment failure because of the development of ADV resistance. Recent clinical observations reported that the cumulative probability of ADV resistance was 3\% of patients after 2 years of therapy (Delaney, 2007). Therefore, developing a rapid, simple and reliable method to detect the ADV-resistant mutations as early as possible is of great clinical importance. LIPA, based on genotypic resistance detecting for genotypes A-H, is considered to be a powerful tool for such use, but sometimes too many probe lines make interpretation difficult, especially for certain codon sites located closely.

In this study, we made some improvements in the RDB assay, resulting in the detection of ADV resistance. First, probes detecting ADV resistance were designed only for genotypes B, C and D, which are the most predominant genotypes in China. This simplified the evaluation of the results, particularly for use in China. Second, PCR products for hybridization were digested into single strand by the exonuclease instead of traditional denaturation, the signal was stronger due to more single strand participating in hybridization. Since a critical requirement in the RDB mutation detection system is that the hybridization condition for all the mutations on the strip should be identical (Chan et al., 1999), different hybridization factors such as probe length, hybridization temperature and time were investigated to establish the optimization conditions.

In the present study, our improved RDB was simple and rapid in detecting the $\mathrm{rtA} 181 \mathrm{~V} / \mathrm{T}$ and $\mathrm{rtN} 236 \mathrm{~T}$ mutations associated with resistance to ADV, especially for genotypes B, C and D, although no genotype D was found in this batch of clinical samples. In addition, there was no clear association between HBV genotype and clinical response to ADV 
therapy, which was consistent with other reports (Moskovitz et al., 2005). Although there are still conflicting reports about this relationship, Fung et al. (2006) have suggested that HBV genotype D is associated with an increased risk of ADV resistance. Therefore, more clinical samples are required to investigate this relationship.

\section{ACKNOWLEDGMENTS}

Research supported by the National 863 Program of Ministry of Science and Technology (\#2008AA02Z424), National Sciences Foundation of China (\#81101310, \#30800972, \#30800970) and Sciences Foundation of Chongqing Education Committee (\#KJ100309). We also thank Dr. Xinhe Huang (University of Kentucky College of Medicine) for critically reviewing and revising the manuscript.

\section{REFERENCES}

Aloman C and Wands JR (2003). Resistance of HBV to adefovir dipivoxil: a case for combination antiviral therapy? Hepatology 38: 1584-1587.

Chan V, Yam I, Chen FE and Chan TK (1999). A reverse dot-blot method for rapid detection of non-deletion alpha thalassaemia. Br. J. Haematol. 104: 513-515.

Dai CY, Chuang WL, Hsieh MY and Lee LP (2007). Adefovir dipivoxil treatment of lamivudine-resistant chronic hepatitis B. Antiviral Res. 75: 146-151.

Delaney WE (2007). Progress in the treatment of chronic hepatitis B: long-term experience with adefovir dipivoxil. $J$. Antimicrob. Chemother. 59: 827-832.

Fung SK, Chae HB, Fontana RJ, Conjeevaram H, et al. (2006). Virologic response and resistance to adefovir in patients with chronic hepatitis B. J. Hepatol. 44: 283-290.

Hadziyannis SJ, Tassopoulos NC, Heathcote EJ, Chang TT, et al. (2006). Long-term therapy with adefovir dipivoxil for HBeAg-negative chronic hepatitis B for up to 5 years. Gastroenterology 131: 1743-1751.

Lok AS, Zoulim F, Locarnini S, Bartholomeusz A, et al. (2007). Antiviral drug-resistant HBV: standardization of nomenclature and assays and recommendations for management. Hepatology 46: 254-265.

Lovicu M, Dessi V, Zappu A, De VS, et al. (2003). Efficient strategy for molecular diagnosis of Wilson disease in the sardinian population. Clin. Chem. 49: 496-498.

Luan J, Yuan J, Li X, Jin S, et al. (2009). Multiplex detection of 60 hepatitis B virus variants by maldi-tof mass spectrometry. Clin. Chem. 55: 1503-1509.

Moskovitz DN, Osiowy C, Giles E, Tomlinson G, et al. (2005). Response to long-term lamivudine treatment (up to 5 years) in patients with severe chronic hepatitis B, role of genotype and drug resistance. J. Viral. Hepat. 12: 398-404.

Ohishi W, Shirakawa H, Kawakami Y, Kimura S, et al. (2004). Identification of rare polymerase variants of hepatitis B virus using a two-stage PCR with peptide nucleic acid clamping. J. Med. Virol. 72: 558-565.

Osiowy C, Villeneuve JP, Heathcote EJ, Giles E, et al. (2006). Detection of rtN236T and rtA181V/T mutations associated with resistance to adefovir dipivoxil in samples from patients with chronic hepatitis B virus infection by the INNOLiPA HBV DR line probe assay (version 2). J. Clin. Microbiol. 44: 1994-1997.

Perz JF, Armstrong GL, Farrington LA, Hutin YJ, et al. (2006). The contributions of hepatitis B virus and hepatitis C virus infections to cirrhosis and primary liver cancer worldwide. J. Hepatol. 45: 529-538.

Santantonio T, Fasano M, Durantel S, Barraud L, et al. (2009). Adefovir dipivoxil resistance patterns in patients with lamivudine-resistant chronic hepatitis B. Antivir. Ther. 14: 557-565.

Tran N, Berne R, Chann R, Gauthier M, et al. (2006). European multicenter evaluation of high-density DNA probe arrays for detection of hepatitis B virus resistance mutations and identification of genotypes. J. Clin. Microbiol. 44: 2792-2800.

Villet S, Pichoud C, Billioud G, Barraud L, et al. (2008). Impact of hepatitis B virus rtA181V/T mutants on hepatitis B treatment failure. J. Hepatol. 48: 747-755.

Wang YZ, Xiao JH, Ruan LH, Zhang HY, et al. (2009). Detection of the rtA181V/T and rtN236T mutations associated with resistance to adefovir dipivoxil using a ligase detection reaction assay. Clin. Chim. Acta 408: 70-74.

Zhao Y, Zhang XY, Guo JJ, Zeng AZ, et al. (2010). Simultaneous genotyping and quantification of hepatitis B virus for genotypes B and C by real-time PCR assay. J. Clin. Microbiol. 48: 3690-3697. 